\title{
Energies and Lifetimes of Levels for Doubly Ionized Xenon and Radon
}

\author{
S. ESER* AND L. ÖZDEMIR \\ Department of Physics, Sakarya University, 54187, Sakarya, Turkey \\ (Received January 4, 2018; in final form March 12, 2018)
}

\begin{abstract}
We have reported the energies and radiative lifetimes of levels for doubly ionized xenon (Xe III) and radon (Rn III). The calculations have been performed using the general-purpose relativistic atomic structure package based on fully relativistic multiconfiguration Dirac-Fock method. We have compared the results obtained from this work for (Xe III) with previous works in available literature. For (Rn III), there is no data except a few energy levels. Hence, we have presented new values on the energy levels of (Rn III).
\end{abstract}

DOI: 10.12693/APhysPolA.133.1324

PACS/topics: 31.15.ag, 31.15.aj, 31.15.V-, 31.30.-i

\section{Introduction}

Atomic data, in particular energy spectra data, has great importance for accurate plasma modelling in astrophysics and plasma physics applications [1-3]. In this work, the energy levels and their lifetimes have been investigated doubly ionized xenon $(Z=54)$ and radon $(Z=86)$. These ions have $n s^{2} n p^{4}$ electron ground configuration ( $n=5$ and 6 , for Xe III, and Rn III, respectively). The ground level for these ions is $n p^{43} P_{2}$, and this level is followed by ${ }^{3} P_{1},{ }^{3} P_{0},{ }^{1} D_{2}$ and ${ }^{1} S_{0}$ in the same configuration. Xenon has played an important role in laser development and technique, from the beginning of the laser era to the actual laser research. Due to its rich emission spectrum, xenon is not only an important element in laser research, but also of critical interest in the broader areas of light sources and lamp development [4]. There are some experimental and theoretical works including radiative lifetimes of ${ }^{1} S_{0}$ metastable state which belongs to ground state configuration for Xe III ion. These works include different ion-trap techniques [5-7] and the least-squares fits to observed energy levels [8], and a study on the comparison of results from iontrap techniques and various theoretical methods, MCHF, MCDF, HXR, and HFR [9]. Biémont et al. [10] calculated the energy levels and radiative transitions for states within $5 p^{k}(k=1-5)$ configurations of atoms and ions in the indium-iodine isoelectronic sequence. The lifetimes for excited levels obtained by time-resolved spectroscopy and relativistic Hartree-Fock calculations for the emission characteristics of an ultraviolet-visible pulsed multiionic xenon laser [11], photon-ion spectrometer at PETRA III for measuring multiple photoionization of $\mathrm{Xe}^{q}$ $(q=1-5)$ ions, and beam-foil technique for ionized neonxenon were reported $[12,13]$.

*corresponding author; e-mail: skabakci@sakarya.edu.tr
For doubly ionized xenon, analysis of observations obtained from the atomic spectroscopy works, measurements for radiative lifetimes by the beam-foil spectrum of xenon between 105 and $500 \mathrm{~nm}$, threshold photoelectronthreshold photoelectron coincidence (TPEsCO) spectroscopy and the angular dependence of the UV/VIS and VUV fluorescence on the alignment of Xe II and Xe III ionic states as experimental [14-17]; and by the fourcomponent two-particle propagator technique and an efficient method of inclusion of the core-valence correlations into configuration interaction (CI) calculations as theoretical [18-20] were presented. Garstang reported the results of calculations of the some energy levels and transition probabilities of forbidden lines for a number of atoms and ions of astrophysical or laboratory interest including xenon ions [21]. Using a photon-ion mergedbeam technique, Koizumi et al. presented the experimental results of the $4 d$ photoionization of $\mathrm{Xe}^{q+}(q=1-3)$ and this spectrum was analyzed by multiconfiguration Dirac-Fock calculations [22]. An investigation based on photographic recordings of xenon spectra and the classifications including most of Xe III laser lines were given by Persson et al. [23]. Saloman compiled the energy levels and observed spectral lines of the xenon atom, in all stages of ionization [24].

Radon is a radioactive noble gas element, which is obtained by radioactive disintegration of radium, while all other noble gases are present in atmosphere. The data on energy levels, lifetimes or transition parameters for atomic doubly ionized radon are few in literature. A theoretical study for Rn III was presented by Biémont and Quinet [25].

The aim of this work is to calculate the level energies and lifetimes in doubly ionized xenon and radon, using the general-purpose relativistic atomic structure package (GRASP) [26] based on a fully relativistic multiconfiguration Dirac-Fock (MCDF) method. This code includes the Breit interactions (magnetic interaction between the electrons and retardation effects of the electron-electron interaction) for relativistic effects and quantum electrodynamical (QED) contributions (self-energy and vacuum 
polarization). These contributions are important in investigations including electronic structure and spectroscopic properties of many electron systems. We have taken into account here the configurations including excitations from valence and core (valence, core-valence and core-core correlation) for considering correlation effects. We have selected the configurations of $5 s^{2} 5 p^{4}$, $5 p^{6}, 5 s 5 p^{4} 5 d, 5 s^{2} 5 p^{2} 5 d^{2}, 5 p^{4} 5 d^{2}, 5 s^{2} 5 p^{3} 6 p$ and $5 p^{4} 6 s^{2}$, for even-parity, and $5 s 5 p^{5}, 5 s^{2} 5 p^{3} 5 d, 5 p^{5} 5 d, 5 s^{2} 5 p^{3} 6 s$, $5 s^{2} 5 p^{3} 6 d, 5 s 5 p^{3} 6 s^{2}, 5 s 5 p^{3} 7 s^{2}, 5 p^{5} 6 s$ and $5 s^{2} 5 p^{3} 7 s$, for odd-parity, for Xe III; and $6 s^{2} 6 p^{4}, 6 p^{6}, 6 s^{2} 6 p^{3} 7 p, 6 p^{4} 6 d^{2}$, $6 s^{2} 6 p^{2} 6 d 7 s, 6 p^{4} 6 d 7 s, 6 p^{4} 7 s^{2}$ and $6 s^{2} 6 p^{2} 7 s^{2}$, for evenparity, and $6 s 6 p^{5}, 6 s^{2} 6 p^{3} 7 s, 6 s^{2} 6 p^{3} 6 d, 6 p^{5} 6 d, 6 s 6 p^{3} 7 s^{2}$, $6 s 6 p^{3} 8 s^{2}, 6 p^{5} 7 s, 6 s^{2} 6 p^{3} 7 d$ and $6 s^{2} 6 p^{3} 8 s$, for odd-parity, for Rn III.

\section{Calculation procedure}

The general-purpose relativistic atomic structure package, GRASP code [26] is based on a fully relativistic multiconfiguration Dirac-Fock (MCDF). This code considers the Thomas-Fermi and the Coulomb potential for obtaining the wavefunctions according to JJ and LS coupling. In the MCDF method an atomic state can be expanded as a linear combination of configuration state functions

$$
\Psi_{a}(P J M)=\sum_{r=1}^{n_{c}} C_{r}(\alpha)\left|\gamma_{r}(P J M)\right\rangle
$$

where $n_{c}$ is the number of CSFs included in the evaluation of atomic state functions and $C_{r}$ is the mixing coefficient, optimized usually on the basis of the manyelectron Dirac-Coulomb Hamiltonian. This method is basic and requires no knowledge of the internal coupling of the CSFs with a given parity $P$ and angular momentum $(J, M)$. The CSFs are the sum of products of singleelectron Dirac spinors,

$$
\phi(r, \theta, \varphi, \sigma)=\frac{1}{r}\left(\begin{array}{l}
P(r) \chi_{\kappa m}(\theta, \varphi, \sigma) \\
\mathrm{i} Q(r) \chi_{-\kappa m}(\theta, \varphi, \sigma)
\end{array}\right),
$$

where $\kappa$ is a quantum number and $\chi_{\kappa m}$ is the spinor spherical harmonic in the LSJ coupling scheme. The $P(r)$ and $Q(r)$ are large and small radial components of one-electron wave functions represented on a logarithmic grid. The energy functional is based on the DiracCoulomb Hamiltonian,

$H_{D C}=\sum_{j=1}^{N}\left(\left(C \boldsymbol{\alpha}_{j} \cdot \boldsymbol{p}_{j}\right)+\left(\beta_{j}-1\right) c^{2}+V\left(r_{j}\right)\right)+\sum_{j<k}^{N} \frac{1}{r_{j k}}$,

where $V\left(r_{j}\right)$ is the electron-nucleon interaction. Once initial and final state functions have been calculated, the radiative matrix element for radiative properties computation can be obtained from

$$
O_{i f}=\left\langle\psi(i)\left|\boldsymbol{O}_{q}^{\pi(k)}\right| \psi(f),\right\rangle
$$

where $\boldsymbol{O}_{q}^{\pi(k)}$ is a spherical operator of rank $k$ and parity $\pi$, and $\pi(k)$ is $\pi=(-1)^{k}$, for an electric multipole transition or $\pi=(-1)^{k+1}$, for a magnetic multipole transition. The largest transition probability is for electric dipole
(E1) radiation, dominated by the least factor $1 / \alpha^{2}$ over other types of transitions (E2, M1, M2, etc.).

The transition probabilities for the emission from the upper level to the lower level is given by

$$
\begin{aligned}
& A^{\pi k}\left(\gamma^{\prime} J^{\prime}, \gamma J\right)= \\
& \quad 2 C_{k}\left[\alpha\left(E_{\gamma^{\prime} J^{\prime}}-E_{\gamma J}\right)\right]^{2 k+1} \frac{S^{\pi k}\left(\gamma^{\prime} J^{\prime}, \gamma J\right)}{g_{J^{\prime}}},
\end{aligned}
$$

where $S^{\pi k}$ is line strength,

$$
S^{\pi k}\left(\gamma^{\prime} J^{\prime}, \gamma J\right)=\left|\left\langle\gamma J\left\|\boldsymbol{O}^{\pi(k)}\right\| \gamma^{\prime} J^{\prime}\right\rangle\right|^{2},
$$

$C_{k}=(2 k+1)(k+1) / k((2 k+1) ! !)^{2}$, and $\boldsymbol{O}^{\pi(k)}$ is transition operator. Most experiments yield the lifetime of the upper level. In this case the sum over multipole transitions to all lower lying levels has to be taken. The lifetime, $\tau_{\gamma^{\prime} J^{\prime}}$, of upper level $\gamma^{\prime} J^{\prime}$ is

$$
\tau_{\gamma^{\prime} J^{\prime}}=\frac{1}{\sum_{\pi k, \gamma J} A^{\pi k}\left(\gamma^{\prime} J^{\prime}, \gamma J\right)}
$$

In calculations we have used the option extended average level (EAL) averaging of the expression energy. It is extended to configuration states with not only different total angular momentum but also with different parity. Also, the Breit corrections (magnetic interaction between the electrons and retardation effects of the electron-electron interaction), and QED (self-energy and vacuum polarization), and various correlation contributions have been considered. Due to the Coulomb interaction between the electrons, the electron correlation effects are also important, in particular, for fine structure and transitions. Therefore, the configurations including excitations from valence and core have been taken into account in calculations. QED contributions are self-energy and vacuum polarization, which are also included in the computations of the transition energy. The finite-nucleus effect is taken into account by assuming an extended Fermi distribution for the nucleus. Both the Breit and QED contributions are treated as perturbation and are not included directly in the SCF procedure. The mixing coefficients are calculated by diagonalizing the modified Hamiltonian.

\section{Results}

In this paper, we have presented the calculations of excitation energies and lifetimes for Xe III and Rn III using the (GRASP) code [26]. In the calculations, we have taken into account the various correlation effects (valence-valence, core-valence, and core-core) besides Breit (magnetic interaction between the electrons and retardation effects of the electron-electron interaction), and QED (self-energy and vacuum polarization) corrections. Therefore, we have considered the configurations of $5 s^{2} 5 p^{4}, 5 p^{6}, 5 s 5 p^{4} 5 d, 5 s^{2} 5 p^{2} 5 d^{2}, 5 p^{4} 5 d^{2}, 5 s^{2} 5 p^{3} 6 p$, $5 p^{4} 6 s^{2}$ (for even-parity) and $5 s 5 p^{5}, 5 s^{2} 5 p^{3} 5 \mathrm{~d}, 5 p^{5} 5 d$, $5 s^{2} 5 p^{3} 6 s, 5 s^{2} 5 p^{3} 6 d, 5 s 5 p^{3} 6 s^{2}, 5 s 5 p^{3} 7 s^{2}, 5 p^{5} 6 s, 5 s^{2} 5 p^{3} 7 s$ (for odd-parity), for Xe III; and $6 s^{2} 6 p^{4}, 6 p^{6}, 6 s^{2} 6 p^{3} 7 p$, $6 p^{4} 6 d^{2}, 6 s^{2} 6 p^{2} 6 d 7 s, 6 p^{4} 6 d 7 s, 6 p^{4} 7 s^{2}, 6 s^{2} 6 p^{2} 7 s^{2}$ (for 
even-parity) and $6 s 6 p^{5}, 6 s^{2} 6 p^{3} 7 s, 6 s^{2} 6 p^{3} 6 d, \quad 6 p^{5} 6 d$, $6 s 6 p^{3} 7 s^{2}, 6 s 6 p^{3} 8 s^{2}, 6 p^{5} 7 s, 6 s^{2} 6 p^{3} 7 d, 6 s^{2} 6 p^{3} 8 s$ (for oddparity) for Rn III.

We have reported the energies, $E\left(\mathrm{~cm}^{-1}\right)$ and lifetimes, $\tau$ (s), for Xe III and Rn III in Table I (at the end) and Table II, respectively. In tables, only odd-parity states have been indicated by the superscript "o". Also the number in brackets represents the power of 10 . For doubly ionized xenon (Xe III) we have obtained 453 energy levels. Table I (at the end) lists only 113 energy levels and their radiative lifetimes for Xe III. In addition, the results obtained have been compared with other works in Fig. 1. It is seen that there is an agreement between our results and others. For doubly ionized radon (Rn III), we have obtained 403 energy levels. Table II includes the ground state levels and their lifetimes for comparing with [25]. First 150 energy levels and lifetimes for Rn III have been presented in Table III (at the end). In addition, a comparing between our results and other values for ground state levels have been given in Fig. 2. Therefore we can mention that the values for Rn III in Table III (at the end) have been reported firstly.

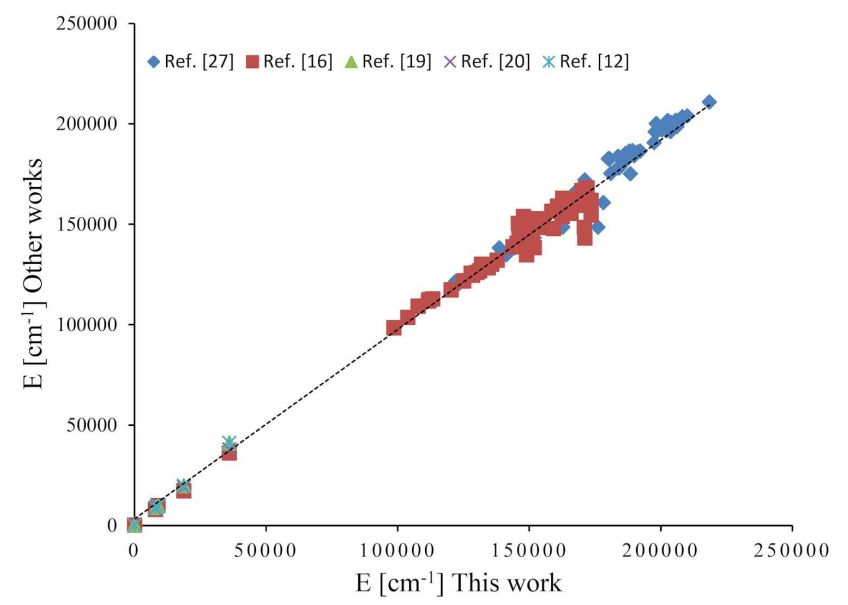

Fig. 1. Comparison of energy levels for Xe III ion with other studies.

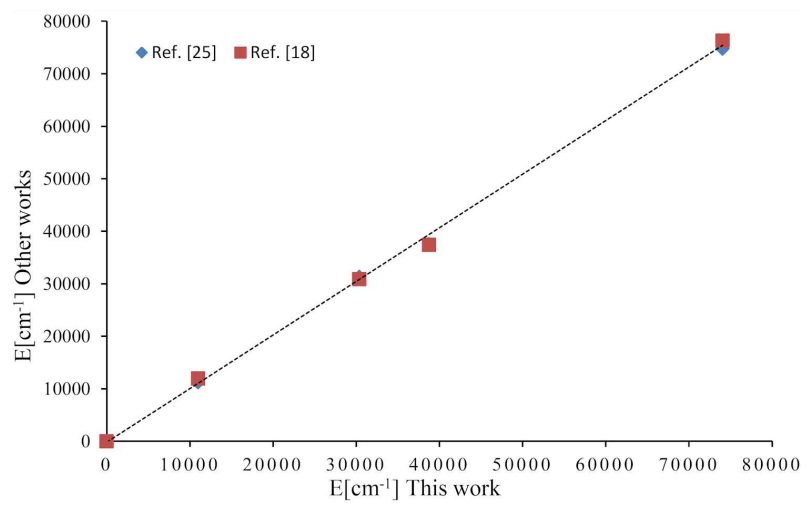

Fig. 2. Comparison of energy levels for Rn III ion with other studies.
TABLE II

Energies $\mathrm{E}\left[\mathrm{cm}^{-1}\right]$ and lifetimes $\tau[\mathrm{s}]$ for the levels of the ground configuration for Rn III. Numbers in brackets represent power of 10 .

\begin{tabular}{c|c|c|c}
\hline \hline \multirow{2}{*}{ Levels } & \multicolumn{2}{|c|}{$E\left[\mathrm{~cm}^{-1}\right]$} & $\tau[\mathrm{s}]$ \\
\cline { 2 - 4 } & this work & other works & this work \\
\hline $6 s^{2} 6 p^{4}{ }^{3} P_{2}$ & 0.000 & 0.000 & \\
$6 s^{2} 6 p^{4}{ }^{3} P_{0}$ & 11002.90 & $11239^{a}$ & $81.96(-3)$ \\
& & $11936.99^{b}$ & \\
$6 s^{2} 6 p^{4}{ }^{3} P_{1}$ & 30385.03 & $31333^{a}$ & $44.44(-7)$ \\
& & $30891.02^{b}$ & \\
$6 s^{2} 6 p^{4}{ }^{1} D_{2}$ & 38765.58 & $37536^{a}$ & $15.72(-7)$ \\
& & $37424.11^{b}$ & \\
$6 s^{2} 6 p^{4}{ }^{1} S_{0}$ & 74050.08 & $74765^{a}$ & $32.57(-8)$ \\
& & $76300.01^{b}$ & \\
\hline$a^{a}$ Ref. [25], ${ }^{b}$ Ref. [18]
\end{tabular}

\section{Conclusion}

The main purpose of this paper is to obtain energy levels and radiative lifetimes for doubly ionized xenon and radon. We have compared our results in available literature and reported new data, in particular Rn III. In this work, the values reported for energy levels and radiative lifetimes can be useful to investigations of some radiative parameters. We hope that these results will be useful for theoretical and experimental works on Xe III and Rn III spectra in future.

\section{Acknowledgments}

The authors are very grateful to the anonymous reviewer for stimulating comments and valuable suggestions, which resulted in improving of the paper.

\section{References}

[1] H. Elabidi, J. Phys. Conf. Ser. 397, 012055 (2012).

[2] P. Rynkun, P. Jönsson, G. Gaigalas, C. Froese Fischer, Astron. Astrophys. A136, 557 (2013).

[3] E. Träbert, Phys. Scr. 61, 257 (2000).

[4] S. Seidel, Th. Wrubel, G. Roston, H.-J. Kunze, J. Quant. Spectrosc. Radiat. Transfer 71, 703 (2001).

[5] K.G. Bhushan, H.B. Pedersen, N. Altstein, O. Heber, M.L. Rappaport, D. Zajfman, Phys. Rev. A 62, 012504 (2000).

[6] A.G. Calamai, C.E. Johnson, Phys. Rev. A 45, 7792 (1992).

[7] R.A. Walch, R.D. Knight, Phys. Rev. A 38, 2375 (1988).

[8] J.E. Hansen, W. Persson, Phys. Scr. 25, 487 (1982).

[9] E. Träbert, Phys. Scr. 85, 048101 (2012).

[10] E. Biémont, J.E. Hansen, P. Quinet, C.J. Zeippen, Astron. Astrophys. Suppl. Ser. 111, 333 (1995).

[11] H. Sobral, M. Raineri, D. Schinca, M. Gallardo, R. Duchowicz, IEEE J. Quantum Electron. 35, 1308 (1999). 
[12] S. Schippers, S. Ricz, T. Buhr, A. Borovik Jr., J. Hellhund, K. Holste, K. Huber, H.-J. Schäfer, D. Schury, S. Klumpp, K. Mertens, M. Martins, R. Flesch, G. Ulrich, E. Rühl, T. Jahnke, J. Lower, D. Metz, L.P.H. Schmidt, M. Schöffler, J.B. Williams, L. Glaser, F. Scholz, J. Seltmann, J. Viefhaus, A. Dorn, A. Wolf, J. Ullrich, A. Müller, J. Phys. B At. Mol. Opt. Phys. 47115602 (2014).

[13] T. Andersen, O.H. Madsen, G. Sørensen, Phys. Scr. 6, 125 (1972)

[14] M. Gallardo, C.A. Massone, A.A. Tagliaferri, M. Garavaglia, W. Persson, Phys. Scr. 19, 538 (1979)

[15] F.J. Coetzer, P. van der Westhuizen, Z. Phys. 294, 199 (1980).

[16] P. Bolognesi, S.J. Cavanagh, L. Avaldi, R. Camilloni, M. Zitnik, M. Stuhec, G.C. King, J. Phys. B At. Mol. Opt. Phys. 33, 4723 (2000).

[17] A. Ehresmann, H. Schäffer, F. Vollweiler, G. Mentzel, B. Magel, K.-H. Schartner, H. Schmoranzer, J. Phys. B At. Mol. Opt. Phys. 31, 1487 (1998).

[18] M. Pernpointner, J.P. Zobel, N.V. Kryzhevoi, Phys. Rev. A 85, 012505 (2012).

[19] M. Pernpointner, J. Phys. B At. Mol. Opt. Phys. 43, 205102 (2010).

[20] V.A. Dzuba, V.V. Flambaum, Phys. Rev. A 75 052504 (2007)
[21] R.H. Garstang, J. Res. Natl. Bur. Stand. Sec. A 68A, 61 (1963).

[22] T. Koizumi, Y. Awaya, A. Fujino, Y. Itoh, M. Kitajima, T.M. Kojima, M. Oura, R. Okuma, M. Sano T. Seikioka, N. Watanabe, F. Koike, Phys. Scr. T73, 131 (1997).

[23] W. Persson, C.-G. Wahlström, G. Bertuccelli, H.O. Di Rocco, J.G. Reyna Almandos, M. Gallardo, Phys. Scr. 38, 347 (1988).

[24] E.B. Saloman, J. Phys. Chem. Ref. Data 33, 765 (2004).

[25] E. Biémont, P. Quinet, Phys. Scr. 54, 36 (1996).

[26] K.G. Dyall, I.P. Grant, C.T. Johnson, F.A. Parpia, E.P. Plummer, Comp. Phys. Commun. 55, 425 (1989).

[27] A. Kramida, Yu. Ralchenko, J. Reader, NIST ASD Team (2015). NIST Atomic Spectra Database (ver. 5.3), National Institute of Standards and Technology, Gaithersburg, MD, Available: 2017, May 18.

TABLE I

Energies, $E\left[\mathrm{~cm}^{-1}\right]$ and lifetimes $\tau[\mathrm{s}]$ of levels for Xe III. Numbers in brackets represent power of 10.

\begin{tabular}{|c|c|c|c|c|}
\hline \multirow{2}{*}{ Levels } & \multicolumn{2}{|c|}{$E\left[\mathrm{~cm}^{-1}\right]$} & \multicolumn{2}{|c|}{$\bar{\tau} \tau[\mathrm{s}]$} \\
\hline & this work & other works & this work & other works \\
\hline $5 s^{2} 5 p^{43} P_{2}$ & 0.0000 & 0.0000 & - & - \\
\hline \multirow[t]{5}{*}{$5 s^{2} 5 p^{4}{ }^{3} P_{0}$} & 7985.62 & $8130.08^{a}$ & $51.54(2)$ & - \\
\hline & & $7952.62^{b}$ & & \\
\hline & & $8929.7^{c}$ & & \\
\hline & & $8313^{d}$ & & \\
\hline & & $8928.5^{e}$ & & \\
\hline \multirow[t]{5}{*}{$5 s^{2} 5 p^{4{ }^{3}} P_{1}$} & 8952.48 & $9794.36^{a}$ & $68.02(-3)$ & $54.40(-3)^{e}$ \\
\hline & & $9751.24^{b}$ & & \\
\hline & & $9657.7^{c}$ & & \\
\hline & & $9638^{d}$ & & \\
\hline & & $9654.45^{e}$ & & \\
\hline \multirow[t]{5}{*}{$5 s^{2} 5 p^{4}{ }^{1} D_{2}$} & 18767.85 & $17098.73^{a}$ & $46.72(-3)$ & $38.00(-3)^{e}$ \\
\hline & & $17163.47^{b}$ & & \\
\hline & & $19689.1^{c}$ & & \\
\hline & & $19086^{d}$ & & \\
\hline & & $19687.98^{e}$ & & \\
\hline \multirow[t]{6}{*}{$5 s^{2} 5 p^{4}{ }^{1} S_{0}$} & 36102.22 & $36102.94^{a}$ & $4.761(-3)$ & $4.10(-3)^{e}$ \\
\hline & & $35964.24^{b}$ & & $4.40(-3)^{f}$ \\
\hline & & $41052.3^{c}$ & & $4.90(-3)^{g}$ \\
\hline & & $37820^{d}$ & & $4.50(-3)^{h}$ \\
\hline & & $41053.59^{e}$ & & $4.60(-3)^{j}$ \\
\hline & & & & $4.46(-3)^{k}$ \\
\hline \multirow[t]{2}{*}{$5 s 5 p^{5}{ }^{3} P_{2}^{o}$} & 98554.00 & $98262.47^{a}$ & $2.87(-8)$ & - \\
\hline & & $98318.93^{b}$ & & \\
\hline \multirow[t]{2}{*}{$5 s 5 p^{5}{ }^{3} P_{1}^{o}$} & 103818.01 & $103568.20^{a}$ & $2.48(-8)$ & - \\
\hline & & $103513.14^{b}$ & & \\
\hline
\end{tabular}


TABLE I (cont.)

\begin{tabular}{|c|c|c|c|c|}
\hline \multirow{2}{*}{ Levels } & \multicolumn{2}{|c|}{$E\left[\mathrm{~cm}^{-1}\right]$} & \multicolumn{2}{|c|}{$\tau[\mathrm{s}]$} \\
\hline & this work & other works & this work & other works \\
\hline $5 s 5 p^{53} P_{0}^{o}$ & 107740.27 & $\begin{array}{l}108333.76^{a} \\
109054.17^{b}\end{array}$ & $9.25(-8)$ & - \\
\hline $5 s^{2} 5 p^{3}\left({ }^{4} S^{o}\right) 5 d^{5} D_{3}^{o}$ & 111686.78 & $\begin{array}{l}111605.41^{a} \\
111707.73^{b}\end{array}$ & $2.35(-8)$ & - \\
\hline $5 s^{2} 5 p^{3}\left({ }^{4} S^{o}\right) 5 d^{5} D_{2}^{o}$ & 112061.34 & $111856.38^{a}$ & $2.18(-8)$ & - \\
\hline $5 s^{2} 5 p^{3}\left({ }^{4} S^{o}\right) 5 d^{5} D_{4}^{o}$ & 112061.10 & $\begin{array}{l}112271.78^{a} \\
112288.45^{b}\end{array}$ & 411.52 & - \\
\hline $5 s^{2} 5 p^{3}\left({ }^{4} S^{o}\right) 5 d^{5} D_{1}^{o}$ & 112693.10 & $112449.90^{a}$ & $16.23(-9)$ & - \\
\hline $5 s^{2} 5 p^{3}\left({ }^{4} S^{o}\right) 5 d^{5} D_{0}^{o}$ & 113293.16 & $\begin{array}{l}112693.95^{a} \\
112675.59^{b}\end{array}$ & $17.95(-9)$ & - \\
\hline $5 s^{2} 5 p^{3}\left({ }^{4} S^{o}\right) 5 d^{3} D_{2}^{o}$ & 120309.23 & $\begin{array}{l}117240.08^{a} \\
117224.56^{b}\end{array}$ & $10.41(-9)$ & - \\
\hline $5 s^{2} 5 p^{3}\left({ }^{4} S^{o}\right) 6 s^{5} S_{2}^{o}$ & 121954.01 & $121475.94^{a}$ & $4.36(-9)$ & - \\
\hline $5 s^{2} 5 p^{3}\left({ }^{2} D^{o}\right) 5 d^{1} P_{1}^{o}$ & 122230.88 & $119026.03^{a}$ & $11.24(-9)$ & - \\
\hline $5 s^{2} 5 p^{3}\left({ }^{4} S^{o}\right) 5 d^{3} D_{3}^{o}$ & 124015.95 & $\begin{array}{l}121229.58^{a} \\
121233.13^{b}\end{array}$ & $14.30(-9)$ & - \\
\hline $5 s^{2} 5 p^{3}\left({ }^{4} S^{o}\right) 5 d^{3} D_{1}^{o}$ & 125106.57 & $\begin{array}{l}121922.75^{a} \\
121725.13^{b}\end{array}$ & $7.75(-9)$ & - \\
\hline $5 s^{2} 5 p^{3}\left({ }^{4} S^{o}\right) 6 s^{3} S_{1}^{o}$ & 128024.81 & $\begin{array}{l}125617.06^{a} \\
125491.74^{b}\end{array}$ & $0.21(-9)$ & - \\
\hline $5 s^{2} 5 p^{3}\left({ }^{2} D^{o}\right) 5 d^{3} F_{2}^{o}$ & 128529.29 & $\begin{array}{l}124691.33^{a} \\
124596.46^{b}\end{array}$ & $12.72(-9)$ & - \\
\hline $5 s^{2} 5 p^{3}\left({ }^{2} D^{o}\right) 5 d^{3} F_{3}^{o}$ & 130139.83 & $\begin{array}{l}126119.77^{a} \\
125967.60^{b}\end{array}$ & $8.20(-9)$ & - \\
\hline $5 s^{2} 5 p^{3}\left({ }^{2} D^{o}\right) 5 d^{1} S_{0}^{o}$ & 131144.30 & $126766.09^{b}$ & $21.05(-8)$ & - \\
\hline $5 s^{2} 5 p^{3}\left({ }^{2} D^{o}\right) 5 d^{3} F_{4}^{o}$ & 131921.73 & $\begin{array}{l}130173.73^{a} \\
129943.91^{b}\end{array}$ & $55.55(-3)$ & - \\
\hline $5 s^{2} 5 p^{3}\left({ }^{2} D^{o}\right) 5 d^{3} G_{3}^{o}$ & 134414.03 & $\begin{array}{l}128349.15^{a} \\
128193.17^{b}\end{array}$ & $3.03(-9)$ & - \\
\hline $5 s^{2} 5 p^{3}\left({ }^{2} D^{o}\right) 5 d^{3} F_{4}^{o}$ & 135700.59 & $\begin{array}{l}130173.73^{a} \\
129943.91^{b}\end{array}$ & $83.33(-3)$ & - \\
\hline $5 s^{2} 5 p^{3}\left({ }^{2} D^{o}\right) 5 d^{3} G_{5}^{o}$ & 137793.96 & $\begin{array}{l}132159.94^{a} \\
132065.15^{b}\end{array}$ & $30.76(-2)$ & - \\
\hline $5 s^{2} 5 p^{3}\left({ }^{2} D^{o}\right) 6 s^{3} D_{1}^{o}$ & 138597.16 & $138145.49^{a}$ & $1.22(-9)$ & $\begin{array}{c}4.60(-9)^{l} \\
4.25(-9)^{l *} \\
4.00(-9)^{m}\end{array}$ \\
\hline $5 s^{2} 5 p^{3}\left({ }^{2} D^{o}\right) 6 s^{3} D_{2}^{o}$ & 138975.58 & $134667.42^{a}$ & $66.2(-10)$ & - \\
\hline $5 s^{2} 5 p^{3}\left({ }^{2} D^{o}\right) 6 s^{3} D_{2}^{o}$ & 141285.68 & $134667.42^{a}$ & $2.69(-9)$ & - \\
\hline $5 s^{2} 5 p^{3}\left({ }^{2} D^{o}\right) 6 s^{3} D_{3}^{o}$ & 143844.05 & $\begin{array}{l}138658.20^{a} \\
138622.44^{b}\end{array}$ & $0.70(-9)$ & - \\
\hline $5 s^{2} 5 p^{3}\left({ }^{2} D^{o}\right) 6 s^{3} D_{1}^{o}$ & 144380.15 & $138145.49^{a}$ & $0.30(-9)$ & - \\
\hline $5 s^{2} 5 p^{3}\left({ }^{2} P^{o}\right) 5 d^{3} P_{0}^{o}$ & 145356.07 & $\begin{array}{l}140437.79^{a} \\
140445.25^{b}\end{array}$ & $1.31(-9)$ & - \\
\hline $5 s^{2} 5 p^{3}\left({ }^{4} S^{o}\right) 6 p^{5} P_{1}$ & 145889.50 & $\begin{array}{l}146781.48^{a} \\
150309.40^{b}\end{array}$ & $2.30(-9)$ & $\begin{array}{c}4.19(-9)^{l} \\
3.34(-9)^{l *} \\
3.40(-9)^{m}\end{array}$ \\
\hline $5 s^{2} 5 p^{3}\left({ }^{4} S^{o}\right) 6 p^{5} P_{2}$ & 146195.85 & $\begin{array}{l}146962.42^{a} \\
146978.34^{b}\end{array}$ & $2.29(-9)$ & $\begin{array}{c}4.32(-9)^{l} \\
3.26(-9)^{l *} \\
3.50(-9)^{m}\end{array}$ \\
\hline $5 s^{2} 5 p^{3}\left({ }^{2} P^{o}\right) 5 d^{3} P_{1}^{o}$ & 146212.06 & $\begin{array}{l}140730.93^{a} \\
140679.15^{b}\end{array}$ & $0.39(-9)$ & - \\
\hline $5 s^{2} 5 p^{3}\left({ }^{2} P^{o}\right) 5 d^{3} D_{2}^{o}$ & 147815.42 & $\begin{array}{l}153893.20^{a} \\
153809.85^{b}\end{array}$ & $0.51(-9)$ & - \\
\hline
\end{tabular}


TABLE I (cont.)

\begin{tabular}{|c|c|c|c|c|}
\hline \multirow{2}{*}{ Levels } & \multicolumn{2}{|c|}{$E\left[\mathrm{~cm}^{-1}\right]$} & \multicolumn{2}{|c|}{$\tau[\mathrm{s}]$} \\
\hline & this work & other works & this work & other works \\
\hline $5 s^{2} 5 p^{3}\left({ }^{4} S^{o}\right) 6 p^{5} P_{3}$ & 147914.95 & $\begin{array}{l}149061.57^{a} \\
149018.92^{b}\end{array}$ & $1.93(-9)$ & $\begin{array}{l}3.21(-9)^{l} \\
2.74(-9)^{l *} \\
3.10(-9)^{m}\end{array}$ \\
\hline $5 s^{2} 5 p^{3}\left({ }^{2} D^{o}\right) 6 s^{1} D_{2}^{o}$ & 148964.22 & $\begin{array}{l}143048.20^{a} \\
134670.32^{b}\end{array}$ & $0.41(-9)$ & - \\
\hline $5 s^{2} 5 p^{3}\left({ }^{2} P^{o}\right) 5 d^{3} F_{3}^{o}$ & 149741.48 & $145340.91^{a}$ & $1.69(-9)$ & - \\
\hline $5 s^{2} 5 p^{3}\left({ }^{4} S^{o}\right) 6 p^{3} P_{1}$ & 150664.93 & $\begin{array}{l}150301.10^{a} \\
150309.40^{b}\end{array}$ & $2.93(-9)$ & $\begin{array}{l}3.22(-9)^{l} \\
4.04(-9)^{l *} \\
2.80(-9)^{m}\end{array}$ \\
\hline $5 s^{2} 5 p^{3}\left({ }^{2} P^{o}\right) 5 d^{3} F_{2}^{o}$ & 151156.64 & $\begin{array}{l}145300.13^{a} \\
145300.70^{b}\end{array}$ & $0.25(-9)$ & - \\
\hline $5 s^{2} 5 p^{3}\left({ }^{2} D^{o}\right) 5 d^{3} D_{3}^{o}$ & 151864.08 & $\begin{array}{l}143156.24^{a} \\
138235.29^{b}\end{array}$ & $0.15(-9)$ & - \\
\hline $5 s^{2} 5 p^{3}\left({ }^{4} S^{o}\right) 6 p^{3} P_{2}$ & 152113.75 & $\begin{array}{l}152057.72^{a} \\
151987.04^{b}\end{array}$ & $3.13(-9)$ & $2.70(-9)^{m}$ \\
\hline $5 s^{2} 5 p^{3}\left({ }^{2} P^{o}\right) 5 d^{3} F_{4}^{o}$ & 152363.49 & $148535.52^{a}$ & $3.53(-3)$ & - \\
\hline $5 s^{2} 5 p^{3}\left({ }^{4} S^{o}\right) 6 p^{3} P_{0}$ & 152722.47 & $\begin{array}{l}152808.17^{a} \\
152704.87^{b}\end{array}$ & $3.34(-9)$ & - \\
\hline $5 s^{2} 5 p^{3}\left({ }^{2} P^{o}\right) 6 s^{3} P^{o} P_{0}$ & 154379.83 & $150505.31^{a}$ & $0.33(-9)$ & - \\
\hline $5 s^{2} 5 p^{3}\left({ }^{2} P^{o}\right) 6 s^{3} P_{1}^{o}$ & 154634.75 & $\begin{array}{l}151482.43^{a} \\
151422.45^{b}\end{array}$ & $0.17(-9)$ & - \\
\hline $5 s^{2} 5 p^{3}\left({ }^{2} P^{o}\right) 5 d^{3} P_{2}^{o}$ & 154582.89 & $\begin{array}{l}150404.24^{a} \\
148373.67^{b}\end{array}$ & $4.00(-9)$ & - \\
\hline $5 s^{2} 5 p^{3}\left({ }^{2} P^{o}\right) 5 d^{3} D_{3}^{o}$ & 158660.18 & $\begin{array}{l}156392.68^{a} \\
156302.10^{b}\end{array}$ & $0.06(-9)$ & - \\
\hline $5 s^{2} 5 p^{3}\left({ }^{2} D^{o}\right) 5 d^{3} S_{1}^{o}$ & 159239.82 & $\begin{array}{l}147797.41^{a} \\
147728.43^{b}\end{array}$ & $0.06(-9)$ & - \\
\hline $5 s^{2} 5 p^{3}\left({ }^{2} P^{o}\right) 6 s^{3} P_{2}^{o}$ & 160733.60 & $\begin{array}{l}158928.10^{a} \\
158955.66^{b}\end{array}$ & $0.10(-9)$ & - \\
\hline $5 s^{2} 5 p^{3}\left({ }^{2} D^{o}\right) 6 p^{3} D_{1}$ & 162097.72 & $158996.98^{a}$ & $2.60(-9)$ & - \\
\hline $5 s^{2} 5 p^{3}\left({ }^{2} P^{o}\right) 5 d^{3} D_{2}^{o}$ & 162527.75 & $\begin{array}{l}153893.20^{a} \\
153809.85^{b}\end{array}$ & $0.05(-9)$ & - \\
\hline $5 s^{2} 5 p^{3}\left({ }^{2} P^{o}\right) 6 s^{1} P_{1}^{o}$ & 162650.47 & $\begin{array}{l}159388.18^{a} \\
159342.81^{b}\end{array}$ & $0.11(-9)$ & - \\
\hline $5 s^{2} 5 p^{3}\left({ }^{2} P^{o}\right) 5 d^{1} F_{3}^{o}$ & 162733.56 & $\begin{array}{l}148412.84^{a} \\
162899.71^{b}\end{array}$ & $29.94(-8)$ & - \\
\hline $5 s^{2} 5 p^{3}\left({ }^{2} D^{o}\right) 6 p^{3} F_{2}$ & 164045.15 & $\begin{array}{l}160691.30^{a} \\
160641.36^{b}\end{array}$ & $2.58(-9)$ & $\begin{array}{c}3.05(-9)^{l} \\
3.25(-9)^{l *}\end{array}$ \\
\hline $5 s^{2} 5 p^{3}\left({ }^{2} D^{o}\right) 6 p^{3} D_{2}$ & 165644.75 & $\begin{array}{l}162259.97^{a} \\
162569.02^{b}\end{array}$ & $2.17(-9)$ & $\begin{array}{l}3.44(-9)^{l} \\
3.34(-9)^{l *}\end{array}$ \\
\hline $5 s^{2} 5 p^{3}\left({ }^{2} P^{o}\right) 5 d^{3} D_{1}^{o}$ & 165818.11 & $\begin{array}{l}155400.90^{a} \\
155310.04^{b}\end{array}$ & $0.005(-9)$ & - \\
\hline $5 s^{2} 5 p^{3}\left({ }^{2} D^{o}\right) 6 p^{3} F_{3}$ & 165995.27 & $\begin{array}{l}162594.81^{a} \\
162569.02^{b}\end{array}$ & $2.23(-9)$ & $\begin{array}{l}3.40(-9)^{l} \\
3.07(-9)^{l *} \\
3.00(-9)^{m}\end{array}$ \\
\hline $5 s^{2} 5 p^{3}\left({ }^{2} P^{o}\right) 6 s^{1} P_{1}^{o}$ & 167879.47 & $\begin{array}{l}159388.18^{a} \\
159342.81^{b}\end{array}$ & $2.308(-9)$ & $0.10(-9)^{n}$ \\
\hline $5 s^{2} 5 p^{3}\left({ }^{2} D^{o}\right) 6 p^{3} D_{3}$ & 168011.78 & $166699.11^{a}$ & $2.90(-9)$ & - \\
\hline $5 s^{2} 5 p^{3}\left({ }^{2} D^{o}\right) 6 p^{3} F_{4}$ & 169946.65 & $\begin{array}{l}166554.82^{a} \\
166601.18^{b}\end{array}$ & $2.23(-9)$ & - \\
\hline $\begin{array}{l}5 s^{2} 5 p^{3}\left({ }^{2} D^{o}\right) 6 p^{3} P_{0} \\
5 s^{2} 5 p^{3}\left({ }^{2} D^{o}\right) 5 d^{3} P_{2}^{o}\end{array}$ & $\begin{array}{l}170362.55 \\
170899.02\end{array}$ & $\begin{array}{l}165941.69^{a} \\
148370.13^{a} \\
148373.67^{b}\end{array}$ & $\begin{array}{c}2.12(-9) \\
0.057(-9)\end{array}$ & - \\
\hline
\end{tabular}


TABLE I (cont.)

\begin{tabular}{|c|c|c|c|c|}
\hline \multirow{2}{*}{ Levels } & \multicolumn{2}{|c|}{$E\left[\mathrm{~cm}^{-1}\right]$} & \multicolumn{2}{|c|}{$\bar{\tau} \tau[\mathrm{s}]$} \\
\hline & this work & other works & this work & other works \\
\hline $5 s^{2} 5 p^{3}\left({ }^{2} D^{o}\right) 6 p^{3} P_{2}$ & 171123.58 & $\begin{array}{l}167066.32^{a} \\
167005.07^{b}\end{array}$ & $2.18(-9)$ & - \\
\hline $5 s^{2} 5 p^{3}\left({ }^{2} D^{o}\right) 6 p^{3} P_{1}$ & 171960.74 & $\begin{array}{l}168086.00^{a} \\
167981.00^{b}\end{array}$ & $2.11(-9)$ & $\begin{array}{c}2.62(-9)^{l} \\
2.62(-9)^{l *}\end{array}$ \\
\hline $5 s^{2} 5 p^{3}\left({ }^{2} D^{o}\right) 5 d^{3} P_{1}^{o}$ & 173478.89 & $\begin{array}{l}154639.37^{a} \\
154455.09^{b}\end{array}$ & $0.042(-9)$ & - \\
\hline $5 s^{2} 5 p^{3}\left({ }^{2} D^{o}\right) 5 d^{1} D_{2}^{o}$ & 173548.53 & $\begin{array}{l}161809.98^{a} \\
161754.40^{b}\end{array}$ & $0.054(-9)$ & - \\
\hline $5 s^{2} 5 p^{3}\left({ }^{2} D^{o}\right) 5 d^{1} F_{3}^{o}$ & 176132.81 & $148412.84^{a}$ & $0.043(-9)$ & - \\
\hline $5 s^{2} 5 p^{3}\left({ }^{2} D^{o}\right) 6 p^{1} D_{2}$ & 171123.58 & $\begin{array}{l}171989.82^{a} \\
143074.61^{b}\end{array}$ & $2.17(-9)$ & $3.3(-9)^{m}$ \\
\hline $5 s^{2} 5 p^{3}\left({ }^{2} D^{o}\right) 5 d^{3} P_{0}^{o}$ & 178135.57 & $160733.77^{a}$ & $0.042(-9)$ & - \\
\hline $5 s^{2} 5 p^{3}\left({ }^{4} S^{o}\right) 6 d^{5} D_{3}^{o}$ & 180083.00 & $182464.48^{a}$ & $1.11(-9)$ & $\begin{array}{l}1.56(-9)^{l} \\
1.78(-9)^{l *}\end{array}$ \\
\hline $5 s^{2} 5 p^{3}\left({ }^{4} S^{o}\right) 6 d^{5} D_{1}^{o}$ & 180097.80 & $182551.32^{a}$ & $0.57(-9)$ & - \\
\hline $5 s^{2} 5 p^{3}\left({ }^{4} S^{o}\right) 6 d^{5} D_{2}^{o}$ & 180128.69 & $182337.88^{a}$ & $1.03(-9)$ & $\begin{array}{c}1.60(-9)^{l} \\
1.72(-9)^{l *}\end{array}$ \\
\hline $5 s^{2} 5 p^{3}\left({ }^{4} S^{o}\right) 6 d^{5} D_{0}^{o}$ & 180142.22 & $182521.94^{a}$ & $0.38(-9)$ & - \\
\hline $5 s^{2} 5 p^{3}\left({ }^{4} S^{o}\right) 6 d^{5} D_{4}^{o}$ & 180215.89 & $182551.32^{a}$ & $1.75(-9)$ & - \\
\hline $5 s^{2} 5 p^{3}\left({ }^{4} S^{o}\right) 7 s^{5} S_{2}^{o}$ & 180548.37 & $182482.74^{a}$ & $1.31(-9)$ & - \\
\hline $5 s^{2} 5 p^{3}\left({ }^{2} P^{o}\right) 6 p^{3} D_{1}$ & 180969.40 & $175231.15^{a}$ & 1.98 & - \\
\hline $5 s^{2} 5 p^{3}\left({ }^{2} P^{o}\right) 6 p^{3} D_{2}$ & 183611.96 & $177955.93^{a}$ & $1.66(-9)$ & $\begin{array}{c}6.99(-9)^{l} \\
6.06(-9)^{l *}\end{array}$ \\
\hline $5 s^{2} 5 p^{3}\left({ }^{2} P^{o}\right) 6 p^{3} P_{1}$ & 183710.42 & $178029.33^{a}$ & $1.68(-9)$ & $4.33(-9)^{l}$ \\
\hline $5 s^{2} 5 p^{3}\left({ }^{4} S^{o}\right) 7 s^{3} S_{1}^{o}$ & 183788.26 & $183786.24^{a}$ & $0.52(-9)$ & - \\
\hline $5 s^{2} 5 p^{3}\left({ }^{2} P^{o}\right) 6 p^{3} P_{0}$ & 184244.92 & $178054.53^{a}$ & $1.82(-9)$ & - \\
\hline $5 s^{2} 5 p^{3}\left({ }^{4} S^{o}\right) 6 d^{3} D_{2}^{o}$ & 186537.05 & $185120.90^{a}$ & $0.15(-9)$ & - \\
\hline $5 s^{2} 5 p^{3}\left({ }^{2} P^{o}\right) 6 p^{3} S_{1}$ & 186935.89 & $182134.14^{a}$ & $1.56(-9)$ & $4.40(-9)^{l}$ \\
\hline $5 s^{2} 5 p^{3}\left({ }^{4} S^{o}\right) 6 d^{3} D_{3}^{o}$ & 187988.95 & $186384.04^{a}$ & $0.14(-9)$ & - \\
\hline $5 s^{2} 5 p^{3}\left({ }^{2} P^{o}\right) 5 d^{1} P_{1}^{o}$ & 188442.18 & $175052.36^{a}$ & $0.052(-9)$ & - \\
\hline $5 s^{2} 5 p^{3}\left({ }^{4} S^{o}\right) 6 d^{3} D_{1}^{o}$ & 189278.19 & $186589.15^{a}$ & $0.072(-9)$ & - \\
\hline $5 s^{2} 5 p^{3}\left({ }^{2} P^{o}\right) 6 p^{1} D_{2}$ & 189897.17 & $184009.10^{a}$ & $2.15(-9)$ & $3.10(-9)^{n}$ \\
\hline $5 s^{2} 5 p^{3}\left({ }^{2} P^{o}\right) 6 p^{1} P_{1}$ & 191175.76 & $185888.03^{a}$ & $2.00(-9)$ & - \\
\hline $5 s^{2} 5 p^{3}\left({ }^{2} P^{o}\right) 6 p^{3} P_{2}$ & 192110.64 & $186320.88^{a}$ & $1.82(-9)$ & - \\
\hline $5 s^{2} 5 p^{3}\left({ }^{2} P^{o}\right) 6 p^{1} S_{0}$ & 197423.20 & $190491.16^{a}$ & $2.20(-9)$ & - \\
\hline $5 s^{2} 5 p^{3}\left({ }^{2} D^{o}\right) 6 d^{3} F_{2}^{o}$ & 197820.23 & $195977.67^{a}$ & $0.66(-9)$ & - \\
\hline $5 s^{2} 5 p^{3}\left({ }^{2} D^{o}\right) 6 d^{3} G_{3}^{o}$ & 198224.73 & $196261.50^{a}$ & $1.31(-9)$ & - \\
\hline $5 s^{2} 5 p^{3}\left({ }^{2} D^{o}\right) 6 d^{3} G_{4}^{o}$ & 198286.10 & $200050.60^{a}$ & $1.84(-9)$ & - \\
\hline $5 s^{2} 5 p^{3}\left({ }^{2} D^{o}\right) 7 s^{3} D_{1}^{o}$ & 198462.27 & $195907.04^{a}$ & $0.55(-9)$ & - \\
\hline $5 s^{2} 5 p^{3}\left({ }^{2} D^{o}\right) 6 d^{1} S_{0}^{o}$ & 198804.05 & $197090.86^{a}$ & $1.01(-9)$ & - \\
\hline $5 s^{2} 5 p^{3}\left({ }^{2} D^{o}\right) 6 d^{3} F_{3}^{o}$ & 198930.14 & $196608.91^{a}$ & $0.45(-9)$ & - \\
\hline $5 s^{2} 5 p^{3}\left({ }^{2} D^{o}\right) 6 d^{3} D_{1}^{o}$ & 200438.25 & $196876.63^{a}$ & $0.23(-9)$ & - \\
\hline $5 s^{2} 5 p^{3}\left({ }^{2} D^{o}\right) 6 d^{3} F_{4}^{o}$ & 201736.54 & $200425.68^{a}$ & $1.73(-9)$ & - \\
\hline $5 s^{2} 5 p^{3}\left({ }^{2} D^{o}\right) 6 d^{3} G_{5}^{o}$ & 202177.17 & $200471.83^{a}$ & $1.80(-9)$ & - \\
\hline $5 s^{2} 5 p^{3}\left({ }^{2} D^{o}\right) 6 d^{1} G_{4}^{o}$ & 202240.04 & $196538.07^{a}$ & $0.59(-9)$ & - \\
\hline $5 s^{2} 5 p^{3}\left({ }^{2} D^{o}\right) 6 d^{3} D_{2}^{o}$ & 202559.25 & $201512.20^{a}$ & $0.24(-9)$ & - \\
\hline $5 s^{2} 5 p^{3}\left({ }^{2} D^{o}\right) 7 s^{3} D_{3}^{o}$ & 202563.90 & $200033.45^{a}$ & $1.18(-9)$ & - \\
\hline $5 s^{2} 5 p^{3}\left({ }^{2} D^{o}\right) 6 d^{3} S_{1}^{o}$ & 203241.91 & $199104.12^{a}$ & $0.17(-9)$ & - \\
\hline $5 s^{2} 5 p^{3}\left({ }^{2} D^{o}\right) 7 s^{3} D_{2}^{o}$ & 203735.59 & $196140.93^{a}$ & $0.28(-9)$ & - \\
\hline $5 s^{2} 5 p^{3}\left({ }^{2} D^{o}\right) 6 d^{3} D_{3}^{o}$ & 203962.10 & $200650.23^{a}$ & $0.17(-9)$ & - \\
\hline $5 s^{2} 5 p^{3}\left({ }^{2} D^{o}\right) 6 d^{3} P_{0}^{o}$ & 205743.51 & $201618.48^{a}$ & $0.13(-9)$ & - \\
\hline $5 s^{2} 5 p^{3}\left({ }^{2} D^{o}\right) 6 d^{3} P_{2}^{o}$ & 206139.06 & $198491.98^{a}$ & $0.11(-9)$ & - \\
\hline $5 s^{2} 5 p^{3}\left({ }^{2} D^{o}\right) 6 d^{3} P_{1}^{o}$ & 207002.74 & $202035.68^{a}$ & $0.10(-9)$ & - \\
\hline
\end{tabular}


TABLE I (cont.)

\begin{tabular}{c|c|c|c|c}
\hline \hline \multirow{2}{*}{ Levels } & \multicolumn{2}{|c|}{$E\left[\mathrm{~cm}^{-1}\right]$} & \multicolumn{2}{c}{$\tau[\mathrm{s}]$} \\
\cline { 2 - 5 } & this work & other works & this work & other works \\
\hline $5 s^{2} 5 p^{3}\left({ }^{2} D^{o}\right) 6 d^{1} D_{2}^{o}$ & 208172.24 & $203376.04^{a}$ & $0.15(-9)$ & - \\
$\left.5 s^{2} 5 p^{3}{ }^{2} D^{o}\right) 6 d^{1} P_{1}^{o}$ & 208289.92 & $202805.90^{a}$ & $0.08(-9)$ & - \\
$5 s^{2} 5 p^{3}\left({ }^{2} D^{o}\right) 6 d^{1} F_{3}^{o}$ & 210089.25 & $203845.36^{a}$ & $0.12(-9)$ & - \\
$5 s^{2} 5 p^{3}\left({ }^{2} P^{o}\right) 6 d^{3} D_{1}^{o}$ & 218430.10 & $210819.29^{a}$ & $0.17(-9)$ & - \\
\hline
\end{tabular}

${ }^{a}$ Ref. [27], ${ }^{b}$ Ref. [16], ${ }^{c}$ Ref. [19], ${ }^{d}$ Ref. [20], ${ }^{e}$ Ref. [12], ${ }^{f}$ Ref. [21], ${ }^{g}$ Ref. [8], ${ }^{h}$ Ref. [7], ${ }^{j}$ Ref. [6], ${ }^{k}$

Ref. [5], ${ }^{l}$ Ref. [15] (exp.), ${ }^{l *}$ Ref. [15] (calc.), ${ }^{m}$ Ref. [13], ${ }^{n}$ Ref. [11]

Note:l and $l^{*}$ values are taken from the same article. There are several $l^{*}$ measurement values corresponding to the theoretical $l$ value. Here, the average value of these measured values is given.

TABLE III

TABLE III (cont.)

Energies $E\left[\mathrm{~cm}^{-1}\right]$ and lifetimes $\tau[\mathrm{s}]$ of the excited levels for Rn III. Numbers in brackets represent power of 10 .

\begin{tabular}{|c|c|c|}
\hline Levels & $E\left[\mathrm{~cm}^{-1}\right]$ & $\overline{\tau \tau[\mathrm{s}]}$ \\
\hline $6 s^{2} 6 p^{3}\left({ }^{4} S^{o}\right) 7 s^{5} S_{2}^{o}$ & 95489.17 & $1.02(-9)$ \\
\hline $6 s^{2} 6 p^{3}\left({ }^{4} S^{o}\right) 7 s^{3} S_{1}^{o}$ & 97602.90 & $0.55(-9)$ \\
\hline $6 s^{2} 6 p^{3}\left({ }^{4} S^{o}\right) 6 d^{5} D_{2}^{o}$ & 97730.77 & $4.19(-9)$ \\
\hline $6 s^{2} 6 p^{3}\left({ }^{4} S^{o}\right) 6 d^{5} D_{3}^{o}$ & 99024.86 & $4.05(-9)$ \\
\hline $6 s^{2} 6 p^{3}\left({ }^{4} S^{o}\right) 6 d^{5} D_{1}^{o}$ & 99743.68 & $3.52(-9)$ \\
\hline $6 s^{2} 6 p^{3}\left({ }^{4} S^{o}\right) 6 d^{5} D_{0}^{o}$ & 100014.37 & $8.54(-10)$ \\
\hline $6 s^{2} 6 p^{3}\left({ }^{4} S^{o}\right) 6 d^{5} D_{4}^{o}$ & 100410.24 & $8.40(-3)$ \\
\hline $6 s^{2} 6 p^{3}\left({ }^{2} P^{o}\right) 6 d^{3} P_{2}^{o}$ & 101089.71 & $1.81(-9)$ \\
\hline $6 s^{2} 6 p^{3}\left({ }^{2} D^{o}\right) 6 d^{3} G_{3}^{o}$ & 115429.83 & $0.25(-9)$ \\
\hline $6 s^{2} 6 p^{3}\left({ }^{4} S^{o}\right) 6 d^{3} D_{1}^{o}$ & 116105.30 & $0.26(-9)$ \\
\hline $6 s 6 p^{53} P_{2}^{o}$ & 119077.51 & $4.17(-9)$ \\
\hline $6 s^{2} 6 p^{3}\left({ }^{4} S^{o}\right) 7 p^{5} P_{1}$ & 119738.65 & $2.25(-9)$ \\
\hline $6 s^{2} 6 p^{3}\left({ }^{4} S^{o}\right) 7 p^{5} P_{2}$ & 119958.05 & $2.11(-9)$ \\
\hline $6 s^{2} 6 p^{3}\left({ }^{4} S^{o}\right) 6 d^{5} D_{1}^{o}$ & 123290.08 & $1.06(-10)$ \\
\hline $6 s^{2} 6 p^{3}\left({ }^{4} S^{o}\right) 7 s^{5} S_{2}^{o}$ & 124965.98 & $0.97(-9)$ \\
\hline $6 s^{2} 6 p^{3}\left({ }^{4} S^{o}\right) 7 p^{5} P_{3}$ & 126001.88 & $1.74(-9)$ \\
\hline $6 s^{2} 6 p^{3}\left({ }^{4} S^{o}\right) 7 p^{3} P_{1}$ & 126729.03 & $1.77(-9)$ \\
\hline $6 s^{2} 6 p^{3}\left({ }^{2} D^{o}\right) 7 s^{3} D_{1}^{o}$ & 127957.03 & $0.61(-9)$ \\
\hline $6 s^{2} 6 p^{3}\left({ }^{2} D^{o}\right) 6 d^{3} F_{3}^{o}$ & 128333.60 & $0.76(-9)$ \\
\hline $6 s^{2} 6 p^{3}\left({ }^{2} D^{o}\right) 6 d^{1} S_{0}^{o}$ & 128426.97 & $4.44(-9)$ \\
\hline $6 s^{2} 6 p^{3}\left({ }^{4} S^{o}\right) 7 p^{3} P_{2}$ & 129319.61 & $1.90(-9)$ \\
\hline $6 s^{2} 6 p^{3}\left({ }^{2} D^{o}\right) 6 d^{3} F_{2}^{o}$ & 129103.49 & $1.77(-9)$ \\
\hline $6 s^{2} 6 p^{3}\left({ }^{2} D^{o}\right) 6 d^{3} G_{4}^{o}$ & 130197.11 & $1.42(-5)$ \\
\hline $6 s^{2} 6 p^{3}\left({ }^{2} D^{o}\right) 6 d^{3} G_{3}^{o}$ & 131894.60 & $1.88(-10)$ \\
\hline $6 s^{2} 6 p^{3}\left({ }^{4} S^{o}\right) 7 p^{3} P_{0}$ & 133331.33 & $1.07(-9)$ \\
\hline $6 s^{2} 6 p^{3}\left({ }^{2} D^{o}\right) 6 d^{3} P_{1}^{o}$ & 133399.66 & $0.79(-9)$ \\
\hline $6 s^{2} 6 p^{3}\left({ }^{2} D^{o}\right) 7 s^{3} D_{3}^{o}$ & 134094.61 & $0.92(-9)$ \\
\hline $6 s^{2} 6 p^{3}\left({ }^{2} D^{o}\right) 6 d^{3} D_{1}^{o}$ & 134623.87 & $0.54(-9)$ \\
\hline $6 s^{2} 6 p^{3}\left({ }^{2} D^{o}\right) 6 d^{3} P_{0}^{o}$ & 134591.40 & $0.91(-9)$ \\
\hline $6 s^{2} 6 p^{3}\left({ }^{2} D^{o}\right) 7 s^{1} D_{2}^{o}$ & 134748.93 & $1.53(-9)$ \\
\hline $6 s^{2} 6 p^{3}\left({ }^{2} D^{o}\right) 6 d^{3} F_{4}^{o}$ & 136746.41 & $1.45(-6)$ \\
\hline $6 s^{2} 6 p^{3}\left({ }^{2} D^{o}\right) 6 d^{3} G_{5}^{o}$ & 139369.33 & $4.27(-6)$ \\
\hline $6 s^{2} 6 p^{3}\left({ }^{2} D^{o}\right) 6 d^{1} G_{4}^{o}$ & 140301.92 & $4.48(-6)$ \\
\hline $6 s^{2} 6 p^{3}\left({ }^{2} D^{o}\right) 6 d^{3} D_{3}^{o}$ & 145205.00 & $0.07(-9)$ \\
\hline $6 s^{2} 6 p^{3}\left({ }^{2} P^{o}\right) 6 d^{3} F_{2}^{o}$ & 145642.22 & $0.26(-9)$ \\
\hline $6 s^{2} 6 p^{3}\left({ }^{2} P^{o}\right) 7 s^{3} P_{0}^{o}$ & 145929.59 & $1.07(-9)$ \\
\hline $6 s^{2} 6 p^{3}\left({ }^{2} P^{o}\right) 7 s^{3} P_{1}^{o}$ & 146111.16 & $0.23(-9)$ \\
\hline $6 s^{2} 6 p^{3}\left({ }^{4} S^{o}\right) 6 d^{3} D_{2}^{o}$ & 147707.86 & $0.37(-9)$ \\
\hline $6 s^{2} 6 p^{3}\left({ }^{2} D^{o}\right) 6 d^{3} S_{1}^{o}$ & 148240.37 & $0.17(-9)$ \\
\hline
\end{tabular}

\begin{tabular}{|c|c|c|}
\hline Levels & $E\left[\mathrm{~cm}^{-1}\right]$ & $\overline{\tau[\mathrm{s}]}$ \\
\hline $6 s^{2} 6 p^{3}\left({ }^{4} S^{o}\right) 7 p^{5} P_{1}$ & 148672.02 & $3.57(-9)$ \\
\hline $6 s^{2} 6 p^{3}\left({ }^{2} D^{o}\right) 7 p^{3} F_{2}$ & 150336.76 & $4.06(-9)$ \\
\hline $6 s^{2} 6 p^{3}\left({ }^{4} S^{o}\right) 7 d^{3} D_{2}^{o}$ & 151867.33 & $0.13(-9)$ \\
\hline $6 s^{2} 6 p^{3}\left({ }^{2} P^{o}\right) 6 d^{3} D_{3}^{o}$ & 152387.58 & $2.61(-9)$ \\
\hline $6 s^{2} 6 p^{3}\left({ }^{4} S^{o}\right) 8 s^{5} S_{2}^{o}$ & 153253.92 & $0.27(-9)$ \\
\hline $6 s^{2} 6 p^{3}\left({ }^{4} S^{o}\right) 8 s^{5} S_{2}^{o}$ & 155220.36 & $0.41(-9)$ \\
\hline $6 s^{2} 6 p^{3}\left({ }^{2} D^{o}\right) 7 p^{3} F_{3}$ & 155169.78 & $2.17(-9)$ \\
\hline $6 s^{2} 6 p^{3}\left({ }^{4} S^{o}\right) 7 d^{3} D_{1}^{o}$ & 155564.58 & $0.22(-9)$ \\
\hline $6 s^{2} 6 p^{3}\left({ }^{2} D^{o}\right) 7 p^{3} D_{2}$ & 155275.15 & $2.08(-9)$ \\
\hline $6 s^{2} 6 p^{3}\left({ }^{4} S^{o}\right) 7 d^{5} D_{3}^{o}$ & 155949.42 & $0.44(-9)$ \\
\hline $6 s^{2} 6 p^{3}\left({ }^{4} S^{o}\right) 7 d^{5} D_{1}^{o}$ & 156526.31 & $0.69(-9)$ \\
\hline $6 s^{2} 6 p^{3}\left({ }^{4} S^{o}\right) 7 d^{5} D_{0}^{o}$ & 156511.05 & $1.78(-9)$ \\
\hline $6 s^{2} 6 p^{3}\left({ }^{4} S^{o}\right) 7 d^{5} D_{2}^{o}$ & 156743.91 & $0.46(-9)$ \\
\hline $6 s^{2} 6 p^{3}\left({ }^{2} D^{o}\right) 7 p^{3} P_{0}$ & 156587.91 & $2.19(-9)$ \\
\hline $6 s^{2} 6 p^{3}\left({ }^{4} S^{o}\right) 7 d^{5} D_{4}^{o}$ & 157029.02 & $1.98(-9)$ \\
\hline $6 s^{2} 6 p^{3}\left({ }^{4} S^{o}\right) 8 s^{3} S_{1}^{o}$ & 157132.48 & $0.38(-9)$ \\
\hline $6 s^{2} 6 p^{3}\left({ }^{2} D^{o}\right) 7 p^{1} F_{3}$ & 157508.49 & $4.45(-9)$ \\
\hline $6 s^{2} 6 p^{3}\left({ }^{2} P^{o}\right) 6 d^{3} P_{0}^{o}$ & 158618.45 & $1.21(-9)$ \\
\hline $6 s^{2} 6 p^{3}\left({ }^{2} D^{o}\right) 6 d^{1} F_{3}^{o}$ & 159108.51 & $0.19(-9)$ \\
\hline $6 s^{2} 6 p^{3}\left({ }^{2} D^{o}\right) 7 p^{3} D_{1}$ & 159157.37 & $2.36(-9)$ \\
\hline $6 s^{2} 6 p^{3}\left({ }^{2} D^{o}\right) 7 p^{3} P_{2}$ & 160922.40 & $2.82(-9)$ \\
\hline $6 s^{2} 6 p^{3}\left({ }^{2} D^{o}\right) 6 d^{1} D_{2}^{o}$ & 163861.35 & $0.07(-9)$ \\
\hline $6 s^{2} 6 p^{3}\left({ }^{2} D^{o}\right) 7 p^{3} D_{3}$ & 163741.14 & $2.18(-9)$ \\
\hline $6 s^{2} 6 p^{3}\left({ }^{2} D^{o}\right) 7 p^{3} F_{4}$ & 163772.82 & $2.24(-9)$ \\
\hline $6 s^{2} 6 p^{3}\left({ }^{2} D^{o}\right) 6 d^{3} S_{1}^{o}$ & 164217.06 & $0.06(-9)$ \\
\hline $6 s^{2} 6 p^{3}\left({ }^{4} S^{o}\right) 7 d^{3} D_{3}^{o}$ & 164836.91 & $0.08(-9)$ \\
\hline $6 s^{2} 6 p^{3}\left({ }^{2} D^{o}\right) 7 p^{3} P_{1}$ & 164951.64 & $2.25(-9)$ \\
\hline $6 s^{2} 6 p^{3}\left({ }^{2} D^{o}\right) 6 d^{3} P_{2}^{o}$ & 168392.69 & $0.04(-9)$ \\
\hline $6 s^{2} 6 p^{3}\left({ }^{2} D^{o}\right) 7 p^{1} D_{2}$ & 169696.71 & $2.66(-9)$ \\
\hline $6 s^{2} 6 p^{3}\left({ }^{2} P^{o}\right) 7 p^{3} D_{1}$ & 170119.66 & $3.03(-9)$ \\
\hline $6 s^{2} 6 p^{3}\left({ }^{2} P^{o}\right) 6 d^{1} P_{1}^{o}$ & 171282.36 & $0.06(-9)$ \\
\hline $6 s^{2} 6 p^{3}\left({ }^{2} P^{o}\right) 7 s^{3} P_{2}^{o}$ & 172328.19 & $0.19(-9)$ \\
\hline $6 s^{2} 6 p^{3}\left({ }^{2} P^{o}\right) 7 p^{3} P_{0}$ & 174261.37 & $2.49(-9)$ \\
\hline $6 s^{2} 6 p^{3}\left({ }^{2} P^{o}\right) 6 d^{3} F_{4}^{o}$ & 175110.44 & $6.63(-7)$ \\
\hline $6 s^{2} 6 p^{3}\left({ }^{2} P^{o}\right) 7 p^{3} P_{1}$ & 176478.28 & $1.75(-9)$ \\
\hline $6 s^{2} 6 p^{3}\left({ }^{2} P^{o}\right) 7 s^{1} P_{1}^{o}$ & 176397.27 & $0.15(-9)$ \\
\hline $6 s^{2} 6 p^{3}\left({ }^{2} P^{o}\right) 7 p^{3} D_{2}$ & 176783.01 & $1.92(-9)$ \\
\hline $6 s^{2} 6 p^{3}\left({ }^{2} P^{o}\right) 6 d^{3} P_{1}^{o}$ & 177788.39 & $0.05(-9)$ \\
\hline $6 s^{2} 6 p^{3}\left({ }^{2} P^{o}\right) 6 d^{3} P_{2}^{o}$ & 179906.13 & $3.70(-9)$ \\
\hline $6 s^{2} 6 p^{3}\left({ }^{4} S^{o}\right) 8 s^{5} S_{2}^{o}$ & 183176.22 & $0.48(-9)$ \\
\hline $6 s^{2} 6 p^{3}\left({ }^{2} P^{o}\right) 6 d^{3} D_{3}^{o}$ & 182948.07 & $0.50(-9)$ \\
\hline
\end{tabular}


TABLE III (cont.)

TABLE III (cont.)

\begin{tabular}{|c|c|c|}
\hline Levels & $\bar{E} E\left[\mathrm{~cm}^{-1}\right]$ & $\bar{\tau} \tau[\mathrm{s}]$ \\
\hline $6 s^{2} 6 p^{3}\left({ }^{2} P^{o}\right) 6 d^{1} F_{3}^{o}$ & 183407.97 & $0.18(-9)$ \\
\hline $6 s^{2} 6 p^{3}\left({ }^{2} D^{o}\right) 8 s^{3} D_{1}^{o}$ & 184634.69 & $0.37(-9)$ \\
\hline $6 s^{2} 6 p^{3}\left({ }^{2} D^{o}\right) 7 d^{1} D_{2}^{o}$ & 184698.24 & $0.19(-9)$ \\
\hline $6 s^{2} 6 p^{3}\left({ }^{2} D^{o}\right) 7 d^{3} P_{0}^{o}$ & 184936.67 & $0.33(-9)$ \\
\hline $6 s^{2} 6 p^{3}\left({ }^{2} D^{o}\right) 7 d^{1} P_{1}^{o}$ & 185506.36 & $0.41(-9)$ \\
\hline $6 s^{2} 6 p^{3}\left({ }^{4} S^{o}\right) 7 d^{5} D_{4}^{o}$ & 186399.36 & $1.93(-9)$ \\
\hline $6 s^{2} 6 p^{3}\left({ }^{2} D^{o}\right) 7 d^{3} F_{2}^{o}$ & 186804.89 & $0.37(-9)$ \\
\hline $6 s^{2} 6 p^{3}\left({ }^{2} D^{o}\right) 7 d^{3} G_{3}^{o}$ & 187831.29 & $0.31(-9)$ \\
\hline $6 s^{2} 6 p^{3}\left({ }^{2} D^{o}\right) 7 d^{3} D_{1}^{o}$ & 188292.85 & $0.14(-9)$ \\
\hline $6 s^{2} 6 p^{3}\left({ }^{2} D^{o}\right) 7 d^{3} D_{3}^{o}$ & 188743.76 & $0.20(-9)$ \\
\hline $6 s^{2} 6 p^{3}\left({ }^{2} D^{o}\right) 7 d^{1} S_{0}^{o}$ & 191563.39 & $0.13(-9)$ \\
\hline $6 s^{2} 6 p^{3}\left({ }^{2} D^{o}\right) 8 s^{3} D_{3}^{o}$ & 192308.42 & $1.09(-9)$ \\
\hline $6 s^{2} 6 p^{3}\left({ }^{2} D^{o}\right) 8 s^{1} D_{2}^{o}$ & 192599.69 & $0.71(-9)$ \\
\hline $6 s^{2} 6 p^{3}\left({ }^{2} D^{o}\right) 7 d^{3} F_{4}^{o}$ & 194416.78 & $1.68(-9)$ \\
\hline $6 s^{2} 6 p^{3}\left({ }^{2} D^{o}\right) 7 d^{3} F_{4}^{o}$ & 195013.57 & $1.87(-9)$ \\
\hline $6 s^{2} 6 p^{3}\left({ }^{2} D^{o}\right) 7 d^{3} G_{5}^{o}$ & 195043.98 & $1.92(-9)$ \\
\hline $6 s^{2} 6 p^{3}\left({ }^{2} D^{o}\right) 7 d^{3} D_{3}^{o}$ & 195954.73 & $0.24(-9)$ \\
\hline $6 s^{2} 6 p^{3}\left({ }^{2} D^{o}\right) 7 d^{3} D_{2}^{o}$ & 196151.83 & $0.07(-9)$ \\
\hline $6 s^{2} 6 p^{3}\left({ }^{2} D^{o}\right) 7 d^{3} S_{1}^{o}$ & 196809.97 & $0.10(-9)$ \\
\hline $6 s^{2} 6 p^{3}\left({ }^{2} D^{o}\right) 7 d^{3} D_{2}^{o}$ & 197471.11 & $0.12(-9)$ \\
\hline $6 s^{2} 6 p^{3}\left({ }^{2} D^{o}\right) 7 d^{3} P_{1}^{o}$ & 200114.83 & $0.05(-9)$ \\
\hline $6 s^{2} 6 p^{3}\left({ }^{2} P^{o}\right) 7 p^{3} S_{1}$ & 200114.77 & $1.85(-9)$ \\
\hline $6 s^{2} 6 p^{3}\left({ }^{2} P^{o}\right) 7 p^{1} D_{2}$ & 200911.66 & $2.67(-9)$ \\
\hline $6 s^{2} 6 p^{3}\left({ }^{2} D^{o}\right) 7 d^{1} D_{2}^{o}$ & 202071.35 & $0.11(-9)$ \\
\hline $6 s^{2} 6 p^{3}\left({ }^{2} D^{o}\right) 7 d^{1} F_{3}^{o}$ & 202524.75 & $0.10(-9)$ \\
\hline $6 s^{2} 6 p^{3}\left({ }^{2} P^{o}\right) 6 d^{1} P_{1}^{o}$ & 203136.83 & $0.07(-9)$ \\
\hline $6 s^{2} 6 p^{3}\left({ }^{2} P^{o}\right) 8 s^{3} P_{0}^{o}$ & 204003.97 & $0.17(-9)$ \\
\hline $6 s 6 p^{53} P_{0}^{o}$ & 204607.55 & $0.03(-9)$ \\
\hline $6 s^{2} 6 p^{3}\left({ }^{2} P^{o}\right) 8 s^{3} P_{1}^{o}$ & 204926.98 & $0.16(-9)$ \\
\hline $6 s^{2} 6 p^{3}\left({ }^{2} P^{o}\right) 7 p^{3} D_{3}$ & 204808.03 & $1.58(-9)$ \\
\hline $6 s^{2} 6 p^{3}\left({ }^{2} P^{o}\right) 7 p^{1} P_{1}$ & 205746.44 & $1.77(-9)$ \\
\hline $6 s^{2} 6 p^{2} 6 d 7 s^{5} F_{1}$ & 206895.58 & $0.33(-9)$ \\
\hline $6 s^{2} 6 p^{3}\left({ }^{2} P^{o}\right) 7 d^{3} F_{2}^{o}$ & 206805.37 & $0.37(-9)$ \\
\hline
\end{tabular}

\begin{tabular}{l|c|c}
\hline \hline \multicolumn{1}{c|}{ Levels } & $E\left[\mathrm{~cm}^{-1}\right]$ & $\tau[\mathrm{s}]$ \\
\hline $6 s^{2} 6 p^{3}\left({ }^{2} P^{o}\right) 7 p^{3} P_{2}$ & 207664.84 & $1.86(-9)$ \\
$6 s^{2} 6 p^{3}\left({ }^{2} P^{o}\right) 7 d^{3} P_{2}^{o}$ & 208311.40 & $0.23(-9)$ \\
$6 s^{2} 6 p^{2} 6 d 7 s^{5} F_{2}$ & 208625.17 & $0.29(-9)$ \\
$6 s^{2} 6 p^{3}\left({ }^{2} P^{o}\right) 7 d^{3} F_{3}^{o}$ & 209069.02 & $0.16(-9)$ \\
$6 s^{2} 6 p^{3}\left({ }^{2} P^{o}\right) 7 d^{3} D_{1}^{o}$ & 210072.65 & $0.09(-9)$ \\
$6 s^{2} 6 p^{3}\left({ }^{2} P^{o}\right) 7 p^{1} S_{0}$ & 212250.49 & $1.86(-9)$ \\
$6 s^{2} 6 p^{2}\left({ }^{3} P\right) 7 s^{23} P_{0}$ & 213938.02 & $0.14(-9)$ \\
$6 s^{2} 6 p^{2} 6 d 7 s^{5} D_{3}$ & 214323.95 & $0.16(-9)$ \\
$6 s 6 p^{5}{ }^{1} P_{1}^{o}$ & 214390.47 & $0.02(-9)$ \\
$6 s^{2} 6 p^{2} 6 d 7 s^{3} F_{2}$ & 221121.96 & $0.14(-9)$ \\
$6 s^{2} 6 p^{3}\left({ }^{2} P^{o}\right) 8 s^{3} P_{2}^{o}$ & 233295.57 & $0.99(-9)$ \\
$6 s^{2} 6 p^{3}\left({ }^{2} P^{o}\right) 8 s^{1} P_{1}^{o}$ & 234426.38 & $0.53(-9)$ \\
$6 s^{2} 6 p^{3}\left({ }^{2} P^{o}\right) 7 d^{3} F_{4}^{o}$ & 236235.23 & $1.82(-9)$ \\
$6 s^{2} 6 p^{2} 6 d 7 s^{5} F_{2}$ & 236543.95 & $0.30(-9)$ \\
$6 s^{2} 6 p^{2} 6 d 7 s^{5} D_{1}$ & 236764.37 & $0.29(-9)$ \\
$6 s^{2} 6 p^{2} 6 d 7 s^{5} F_{4}$ & 236848.32 & $0.50(-9)$ \\
$6 s^{2} 6 p^{2} 6 d 7 s^{5} D_{0}$ & 237091.59 & $0.41(-9)$ \\
$6 s^{2} 6 p^{3}\left({ }^{2} P^{o}\right) 7 d^{3} P_{0}^{o}$ & 237069.51 & $0.12(-9)$ \\
$6 s^{2} 6 p^{3}\left({ }^{2} P^{o}\right) 7 d^{3} P_{2}^{o}$ & 237250.07 & $1.72(-9)$ \\
$6 s^{2} 6 p^{2} 6 d 7 s^{5} F_{3}$ & 237521.85 & $0.29(-9)$ \\
$6 s^{2} 6 p^{3}\left({ }^{2} P^{o}\right) 7 d^{3} P_{1}^{o}$ & 237901.67 & $0.14(-9)$ \\
$6 s^{2} 6 p^{3}\left({ }^{2} P^{o}\right) 7 d^{1} F_{3}^{o}$ & 237945.83 & $0.31(-9)$ \\
$6 s^{2} 6 p^{3}\left({ }^{2} P^{o}\right) 7 d^{3} D_{3}^{o}$ & 238275.10 & $0.48(-9)$ \\
$6 s^{2} 6 p^{3}\left({ }^{2} P^{o}\right) 7 d^{3} D_{2}^{o}$ & 238807.76 & $0.25(-9)$ \\
$6 s^{2} 6 p^{2} 6 d 7 s^{3} F_{2}$ & 240282.69 & $0.22(-9)$ \\
$6 s^{2} 6 p^{2} 6 d 7 s^{1} P_{1}$ & 241833.95 & $0.19(-9)$ \\
$6 s^{2} 6 p^{2} 6 d 7 s^{3} F_{4}$ & 242370.01 & $0.40(-9)$ \\
$6 s^{2} 6 p^{2} 6 d 7 s^{5} F_{5}$ & 244261.32 & $0.47(-9)$ \\
$6 s^{2} 6 p^{2}\left({ }^{3} P\right) 7 s^{2} P_{1}$ & 244772.46 & $0.16(-9)$ \\
$6 s^{2} 6 p^{2} 6 d 7 s^{3} D_{3}$ & 244955.55 & $0.16(-9)$ \\
$6 s^{2} 6 p^{3}\left({ }^{2} P^{o}\right) 7 d^{1} P_{1}^{o}$ & 244942.17 & $0.08(-9)$ \\
$6 s^{2} 6 p^{2} 6 d 7 s^{5} D_{2}$ & 245358.84 & $0.17(-9)$ \\
& &
\end{tabular}

